\title{
A PRACTICAL MEASURE OF THE MAXIMUM VENTILATORY CAPACITY IN HEALTH AND DISEASE \\ BY
}

\author{
M. C. S. KENNEDY \\ From the Medical Research Council Silicosis Treatment Centre, Stoke-on-Trent
}

(RECEIVED FOR PUbLICATION MAY 15,1952 )

The assessment of disability in dust diseases of the lung presents a problem of great practical importance.

Available evidence indicates that disturbance of the alveolar respiratory component of pulmonary function is of importance in producing disability only in such diseases as pulmonary sarcoidosis and berylliosis (Ferris, Affeldt, Kriete, and Whittenberger, 1951) where there is a lesion of the alveolar epithelium. Much further work remains to be done before the importance of disturbance of the mixing component of pulmonary function in producing disability can be evaluated. Evidence is now available, however, that the principal cause of disability in dust diseases of the lung is disturbance of the ventilatory function (Baldwin, Cournand, and Richards, 1949 ; Gilson and HughJones, 1953). This is also true of asthma and to a large extent of emphysema.

This paper is concerned with the assessment of the maximum ventilatory capacity, and presents a method of measuring this aspect of pulmonary function which has been used during the past three years in an out-patient clinic, and which has been found simple and practical.

The oldest objective method for assessing the ventilatory capacity of a subject is the simple vital capacity measurement. This, however, is a static measure of volume and is an imperfect and often misleading index of the ventilatory function (Gilson and Hugh-Jones, 1949). To detect changes in the ventilatory capacity of individual subjects from day to day, and to detect differences between individuals, it is essential to measure the maximum ventilatory capacity. Since the work of Hermannsen (1933) the measurement of the maximum breathing capacity (M.B.C.)-here termed the maximum voluntary ventilation (M.V.V.)- has been used extensively, and is now generally accepted as the best single index of the maximum ventilatory capacity (Gray, Barnum, Matheson, and Spies, 1950).
However, in practice, the determination of the M.V.V. presents a number of difficulties. First, it has been found difficult or impossible in many cases to be certain that the patient is giving of his best in the M.V.V. test, especially when such issues as compensation are at stake, and the test may thus be partly a measure of his co-operation. Secondly, the measurement of the M.V.V. in practice is time-consuming and fatiguing both to the patient and to the observer. For these reasons attention has been devoted to finding a simpler measure of the maximum ventilatory capacity.

It has been suggested (Kennedy, 1950) that a man's maximum voluntary ventilation or his maximum breathing capacity might be predictable from the analysis of his vital capacity tracings recorded on a fast-revolving kymograph. The remainder of this paper summarizes the development of this earlier work.

\section{Apparatus}

A simple vital-capacity spirometer almost identical with that described by Gilson and Hugh-Jones (1949) has been used for recording the various respiratory tracings. The volume of the spirometer bell is roughly 6.5 litres. In order to minimize resistance to air-flow, a 2-in. diameter tube was used inside the spirometer and for the rubber connexion to the metal mouthpiece. The same electrical integrating device as described by Gilson and Hugh-Jones was used for measuring the volume of air breathed during voluntary hyperventilation. The spirometer bell was counterbalanced by means of a long, thin cylindrical weight which moved up and down within a hollow metal circular container. A small ink recording pen was fixed to this counter-weight so that movements of the spirometer bell could be recorded on a fastrevolving kymograph. Various speeds were tried, and it was found that a kymograph driven by an electric synchronous motor so that its drum periphery revolved at $1.24 \mathrm{~cm}$. per second gave the most satisfactory result. The drum speed was checked by an electric time-marker, and was found to be uniform 


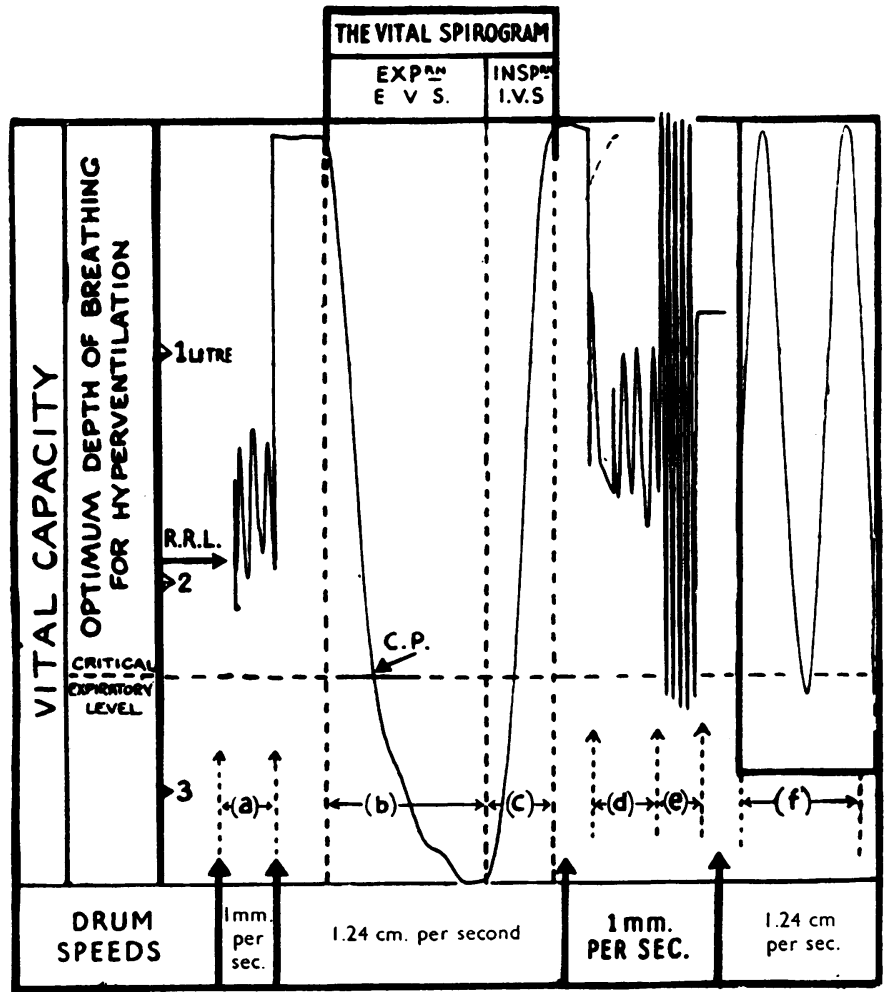

FIG. 1.-A continuous spirometer tracing using different drum speeds of $(a)$ quiet breathing at rest, (b) a forced expiratory vital-capacity tracing or expiratory vital spirogram (E.V.S.), (c) a forced inspiratory vital-capacity tracing or inspiratory vital spirogram (I.V.S.), (d) quiet breathing $+\mathrm{CO}_{2},(e) \mathrm{M}$.V.V. tracing, and vital spirogram (I.V.S.), (d) quiet breathing $+\mathrm{CO}_{2},(e) \mathrm{M} . \mathrm{V} . \mathrm{V}$. tracing, and
$(f)$ sample of an "optimum" M.V.V. tracing. C.P.=the critical point R.R.L. = resting respiratory level.

once the initial inertia had been overcome. In practice the drum was always set in motion a few seconds before recording any tracings to ensure that the drum speed was constant at the time the tracings were recorded.

\section{Procedure}

The following spirographic tracings were obtained as a matter of routine, at any one sitting, on all patients investigated.

Four OR SiX CONSECUTIVE Vital-CAPACITY Tracings.-To obtain each one of these tracings the patient was instructed to take as deep a breath as possible, then insert the mouthpiece, and expel all the air as quickly as possible into the spirometer. The kymograph was set in motion by the observer as soon as he saw the patient about to insert the mouthpiece.

Four Separate Tracings of Maximum Voluntary VENTILATION.-To obtain these tracings the technique of Gilson and Hugh-Jones (1949) has been used. Patients were instructed to hold the mouthpiece firmly between the lips, and then to breathe in and out as deeply and as quickly as possible for 15 seconds. Excessively fast respiratory rates were discouraged for reasons which will be discussed later. The practice of using the mean of three readings as representing the M.V.V. of the patients has in general been adopted. In some of the investigations only the M.V.V. tracing giving the highest value has been analysed. This tracing is termed the optimum M.V.V. The following additional spirographic tracings were obtained on a number of the patients investigated.

Four or Six Consecutive Maximum INSPIRATORY TRACINGS.-For these tracings the patient was instructed to expel all the air in the lungs, then to insert the mouthpiece and inspire as deeply and as quickly as possible.

A Continuous Tracing.-This showed the tidal air at rest, the complementary air, an expiratory vital capacity, an inspiratory vital-capacity tracing, tidal air at rest again, and the maximum breathing capacity. In order to obtain a comparatively compact continuous tracing, different kymograph drum speeds were used for different fractions of the tracing (Fig. 1).

In recording all these tracings a nose clip was worn. It was found that the presence of even a small, single denture could vitiate the tracing ; consequently as a routine all dentures were removed.

\section{Material}

In the course of the past three years some 400 patients have been examined in the manner described above. The majority of these patients were either coal-workers or pottery-workers suffering from pneumoconiosis. The remainder consisted of normal adults and children, and patients with chronic bronchitis and emphysema, asthma, bronchiectasis, neoplasm of the lung, and other pulmonary diseases. The disability of these patients ranged from none to dyspnoea at rest.

Most patients have been examined several times in the manner described, and a few on many occasions, even up to 180 times during the past three years.

\section{FINDINGS}

Definition AND Description OF THE Vital SPIROGRAM.-The vital spirogram (V.S.) is defined as the tracing obtained when a patient expels the 
whole of his vital capacity into a spirometer, and then immediately inspires maximally from the spirometer, the movement of the spirometer being recorded on a fast kymograph. The expiratory phase of the vital spirogram is termed the expiratory vital spirogram (E.V.S.) and the inspiratory phase the inspiratory vital spirogram (I.V.S.). Fig. 1 shows a continuous tracing of quiet breathing at rest, the vital spirogram (V.S.), and maximum voluntary ventilation (M.V.V.).

Study of many hundreds of vital spirograms has shown that in general the I.V.S. is steeper than the E.V.S. and linear throughout its length with the exception of the initial and terminal fractions. The E.V.S. is never uniform throughout its length, and may, except in certain types of abnormal patients, be divided into two fractions. The first fraction is relatively steep and linear. It then breaks away into a second fraction, which is less steep, and later the curve becomes undulatory. The position of the dividing point between the first and second fraction, i.e., where the tracing deviates from a straight line, has been termed the "critical point" (Kennedy, 1950).

In Fig. 1 a dotted line has been drawn through the tracing at the level of the critical point, and it can be seen that it cuts the lower end of the M.V.V. tracing. This has been observed in almost all tracings, and would suggest that this critical level determines the optimum depth of breathing for hyperventilation. Further, the first fraction of the E.V.S. appears to be closely mirrored by the expiratory phase of the M.V.V. tracing $(f)$ in Fig. 1. E.V.S. tracings have been found to be remarkably uniform and almost specific for a given individual, though they may vary from day to day if there is any change in the factors influencing air flow.

The vital spirogram shows at a glance which fraction of the vital capacity is most favourable for hyperventilation. As Cournand, Richards, and Darling (1939) stated, the last fraction of the vital capacity is expelled only with undue effort and expenditure of time, and obviously, if this last fraction is used during forced voluntary ventilation, the minute volume will not be maximal. Rossier, Bucher, and Wiesinger (1947) have suggested that it is desirable for efficient ventilation of the lungs to shift as much air out of and into the lungs as is possible with each breath, since if the volume per breath is reduced to be equal only to the dead-space air, then no useful ventilation will take place at all. Consequently, if the most efficient ventilation is to be achieved, then the greatest volume of air must be shifted per breath in the shortest possible time.
The volume of air shifted during M.V.V. depends on three variables: the rate at which air is expelled, the rate at which air is inspired, and the time lost in changing from inspiration to expiration and vice versa.

THE Hypothesis AND Evolution OF THE METHOD.-A preliminary study of the vital spirogram suggested the hypothesis that it might be possible to assess the M.V.V. from a quantitative analysis of the first fraction of the E.V.S.

In the evolution of the method to be presented, the following questions were first investigated: $(a)$ Is the average flow rate over the first fraction of E.V.S. related to the average expiratory M.V.V. flow rate? Four consecutive E.V.S. tracings and three M.V.V. tracings were obtained from each of 27 patients exhibiting a wide range of M.V.V. values (Fig. 2). The angles $\alpha$, subtended with the horizontal by the first phase of the E.V.S., and

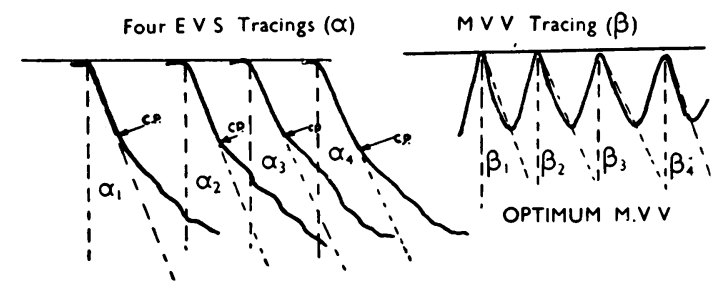

FIG. 2.-The method of comparing the mean slope of line 1 of four E.V.S. tracings $(\alpha)$ with the mean slope of four expiratory phases of an optimum M.V.V. tracing $(\beta)$.

$\beta$, subtended by the mean slope of expiratory phase of the M.V.V. (Fig. 2), were measured. Their tangents are directly proportional to the flow rates during the respective phases of the tracings.

For each value of $\alpha$ the mean of the measurements derived from four consecutive E.V.S. tracings was taken; and similarly for each value of $\beta$, the one of the three M.V.V. tracings which gave the highest or "optimal " value was chosen, and the mean of the measurements derived from four consecutive phases taken.

Fig. 3 shows $\alpha$ plotted against $\beta$, and from this graph it can be seen that the values fall closely about the $45^{\circ}$ slope. Statistically the two sets of means show almost perfect correlation $(r=$ +0.975 ).

(b) What is the relationship between the volume of the first fraction of the E.V.S. and the tidal volume used during the optimum M.V.V.? In the same set of tracings the volumes of the first fraction of the E.V.S. (V) and the tidal volume $\left(V^{1}\right)$ of the optimum M.V.V. were measured (Fig. 4). As before, the values finally taken were the means of measurements derived from four consecutive 


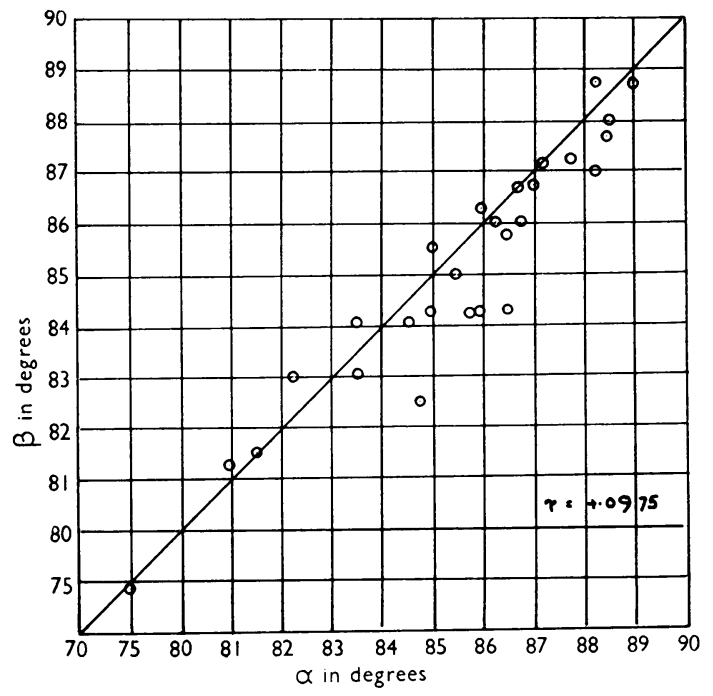

FIG. 3.-The mean slope of line 1 of four E.V.S. tracings $(\alpha)$ plotted against the mean slope of four expiratory phases of an optimum M.V.V. tracing in 27 patients.

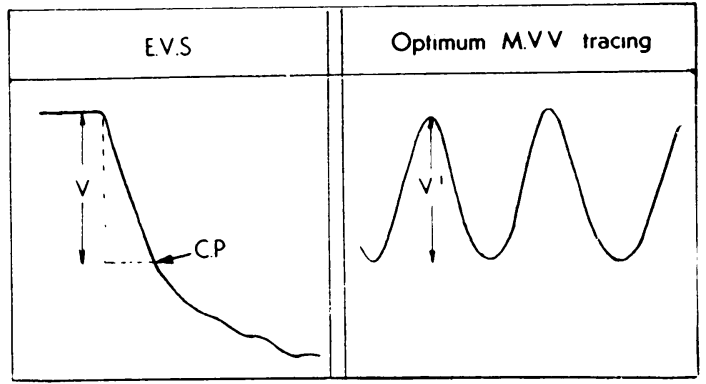

FIG. 4-Correlation between the volume of line $1(\mathrm{~V}=$ depth in $\mathrm{ml}$.) of an E.V.S. and the volume of air shifted per breath when the same patient achieves his optimum M.V.V. (V1).

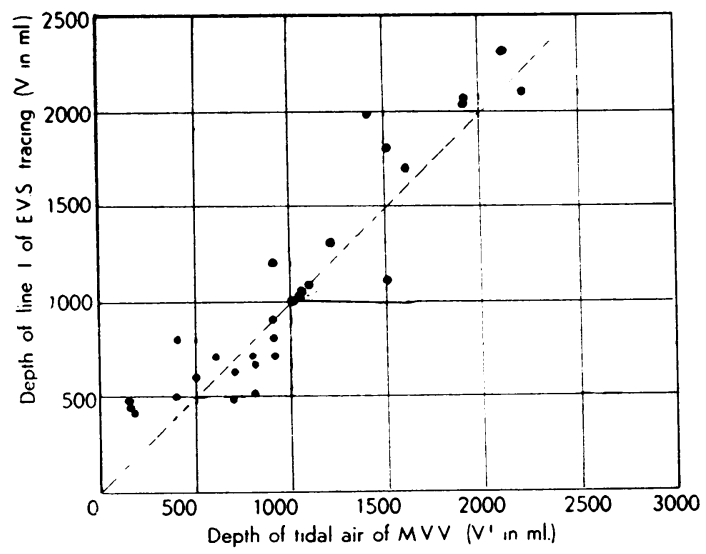

FIG. 5 . tracings of E.V.S. or phases of M.V.V. In Fig. 5 $\mathrm{V}$ and $\mathrm{V}^{1}$ are plotted against each other. In these, as would be expected, there is a considerable deviation on either side of the $45^{\circ}$ slope, but in general $\mathrm{V}^{1}$ increases as $\mathrm{V}$ increases.

(c) Is an average flow rate over the first fraction of the E.V.S. a repeatable measure, and, if so, with what degree of accuracy can this fraction be measured? Four consecutive E.V.S. tracings were obtained from each of 12 patients exhibiting a wide range of M.V.V. values. The angle $\alpha$ (Fig. 2) was measured. The first of the four consecutive E.V.S. tracings given by each of the 12 patients was measured on one day, the second tracing on the next day, and so on, to avoid one reading influencing the observer in measuring the next. These measurements were made independently by two observers.

Statistical analysis of these data gives the following results. (1) The repeatability of E.V.S. tracings on the same man is such that they are distributed with the standard deviation of $0.7^{\circ}$ about their mean. (2) The observer error expressed as the standard error of a single measurement was $0.37^{\circ}$. (3) The measurement of the first fraction of four E.V.S. tracings by one observer is found to give a standard error of $0.40^{\circ}$.

Half the expiratory flow rate (E.F.R.) over the first fraction of E.V.S. may be expressed in litres per minute using the conversion formula $\mathrm{K} \tan \alpha$ =E.F.R. Thus, a man whose mean value for $\alpha$ on his four tracings was $84^{\circ}$ can be said to have a true mean lying between $83.2^{\circ}$ and $84.8^{\circ}$, or, by using the above conversion formula, an E.F.R. between 41.9 and $54.9 \mathrm{l} . / \mathrm{min}$. This range of error will increase and decrease with increasing and decreasing values of $\alpha$.

When $\alpha$ was low an error in reading $\alpha$ of 0.5 would hardly influence the E.F.R. (a change of $75^{\circ}-75.5^{\circ}$ would change the E.F.R. from 18.5 to 19.31 . $/$ min.), but when $\alpha$ approached $90^{\circ}$ then a reading error of $0.5^{\circ}$ might change the E.F.R. value by as much as $501 . / \mathrm{min}$.

In some tracings it was difficult to locate the critical point. In these tracings alternative procedures might be adopted either to measure the volume expired in a fixed time or the time required for a fixed volume. To decide which to choose it is important to answer the following questions:

$(d)$ What is the tidal volume per breath? and $(e)$ What is the rate of breathing when an individual achieves his optimum M.V.V.?

The M.V.V. records of 332 tin-miners and pottery workers were examined. The highest M.V.V. tracing (the optimum M.V.V.) of each 
man was analysed for the respiratory rate and the average tidal volume.

The optimum M.V.V. value of each subject was then plotted against the tidal volume (Fig. 6) used by the subject. The smallest tidal volume recorded during the M.V.V. test was $400 \mathrm{ml}$., and this was observed in five patients who had a mean optimum M.V.V. of $221 . / \mathrm{min}$. From this lowest level the tidal volume is seen to increase as the M.V.V. increases. A tidal volume of $3,900 \mathrm{ml}$. was the highest recorded value (one patient), and the M.V.V. of this patient was $1561 . / \mathrm{min}$. Each group of patients using a given tidal volume has a considerable range of M.V.V. values. However, if the mean M.V.V. of each group is taken the mean M.V.V. value of each group relates well to the tidal volume $(r=+0.946)$ (Fig. 6).

The optimum M.V.V. value of each subject was also plotted against the respiratory rate (Fig. 7). The mean respiratory rate increases with the M.V.V. until an M.V.V. of 60 to $701 . / \mathrm{min}$. is reached. The majority of subjects with an optimum M.V.V. greater than 60 to $701 . / \mathrm{min}$. breathe at a rate of 45 to 60 breaths per min.

The mean values of the optimum M.V.V. and respiratory rate shown in Fig. 7 can be expressed in another way (Fig. 8) to produce an average "optimum" depth rate curve. From this curve one can see at a glance the average depth of breathing and rate of breathing used to produce a given M.V.V. It can be seen that the greater part of

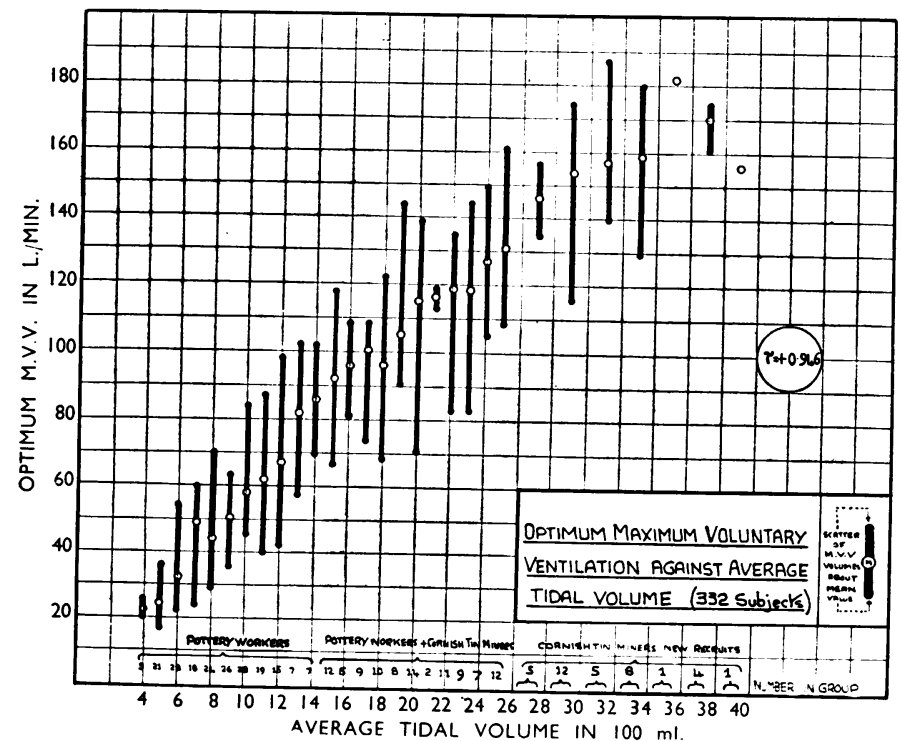

Fig.6.-The optimum M.V.V. of 332 subjects plotted against the tidal volume used in achieving the optimum M.V.V. The subjects were grouped according to their tida! volume.

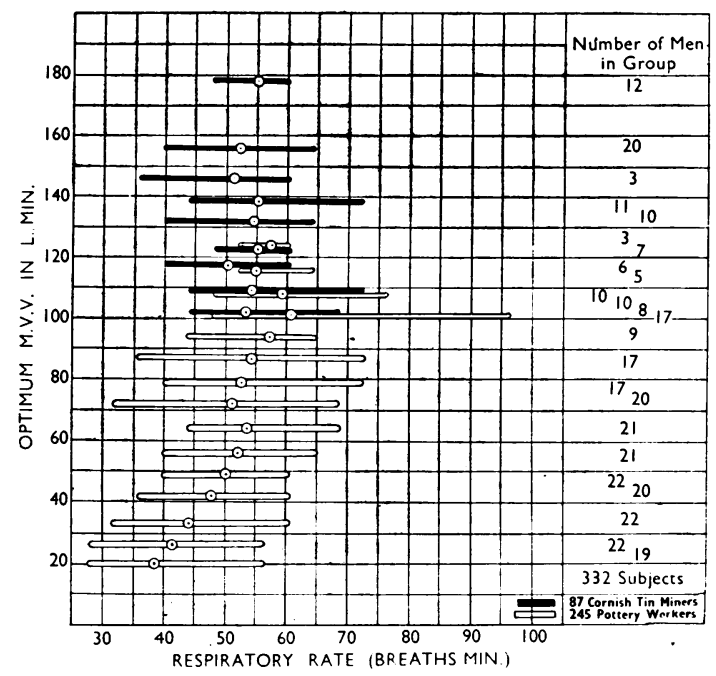

FIG. 7.-The optimum M.V.V. of 332 subjects plotted against the respiratory rate used in achieving the optimum M.V.V. The subjects were grouped according to their M.V.V. The circles indicate the mean M.V.V. of each group, and the lines on either side indicate the range about this mean.

this curve is a straight line, and that it only becomes curved when the M.V.V. falls lower than $601 . / \mathrm{min}$.

Assessment of Maximum Voluntary Ventil.. ATION FROM THE EXPIRATORY Vital SPIROgRaM.This was achieved in two ways.

(1) Using the Average Optimum Depth Rate Line.-If the critical point in the assessment of the E.V.S. is dispensed with, what is required is the optimum depth and rate of breathing for any given individual. This is approximately given by the average "optimum" depth rate curve, since respiratory rate can be converted into expiratory time interval if the approximation is made that the expiratory: inspiratory time interval is $1: 1$. The average "optimum " depth rate curve can be plotted with the volume and time interval coordinates scaled according to the volume excursion of the needle of the spirometer and the speed of the kymograph. If the average optimum depth rate line be so plotted on transparent perspex, then it can be used as the basis of a nomogram for estimating the "optimum" E.F.R of a subject from the E.V.S. tracings. The way in which the E.F.R. is measured using this nomogram is shown in Fig. 9. 


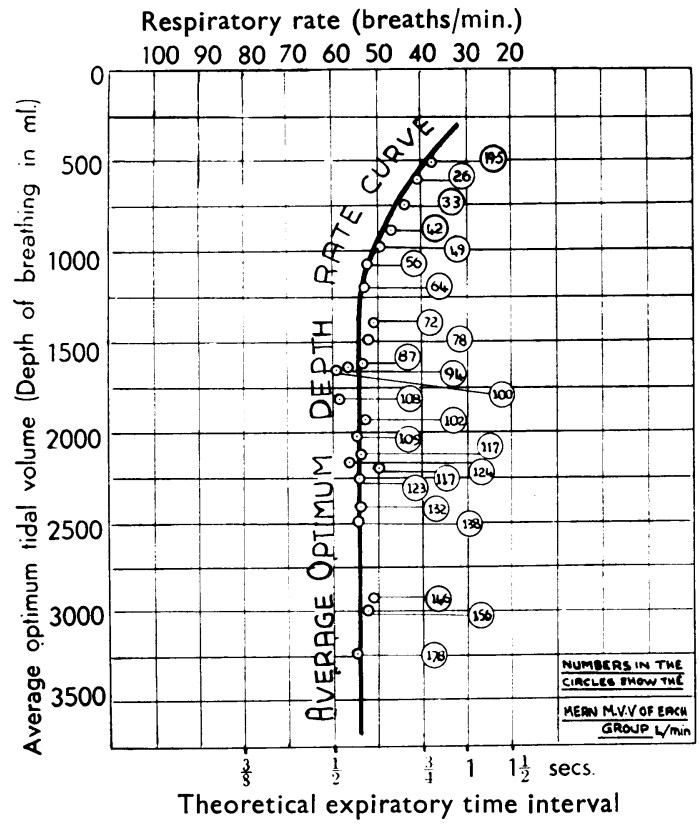

Fig. 8.-The mean M.V.V. values from Fig. 7 have been plotted according to the respiratory rate and tidal volume. The resulting curve shows the rate and depths of breathing that the average
subject will employ to attain his optimum M.V.V. The respirasubject will employ to attain his optimum M.V.V. The respira-
tory rate may also be expressed in seconds as the expiratory time interval if the inspiratory : expiratory time ratio be assumed to be $1: 1$.

The E.F.R. (mean of six E.V.S.) of 50 subjects, covering a wide range of M.V.V. values, was measured using the average optimum depth rate curve. The E.F.R. values were plotted against the mean of three assessments of the M.V.V. (Fig. 10). As can be seen, the E.F.R. values measured in this way agree well with the M.V.V. values $(r=$ +0.853 ).

(2) By Measuring Average Flow Rate Over Two Fixed Periods. - These periods are the first 0.75 sec. period and the first $1.5 \mathrm{sec}$. period of the E.V.S.

As can be seen from Fig. 10, the assessment of flow rates from the E.V.S. using the average optimum depth rate line gives good agreement with the M.V.V. values measured directly, but in practice the nomogram is rather clumsy and timeconsuming to use.

It has already been shown that the M.V.V. is largely influenced by the tidal volume per breath, and that the rate of breathing only varies significantly in those people who have a low M.V.V. Throughout this work a false assumption has been made that the expiratory:inspiratory time interval is $1: 1$. As can be seen from Fig. 11, the majority of subjects whose expiratory time interval is prolonged are subjects who have an M.V.V. of

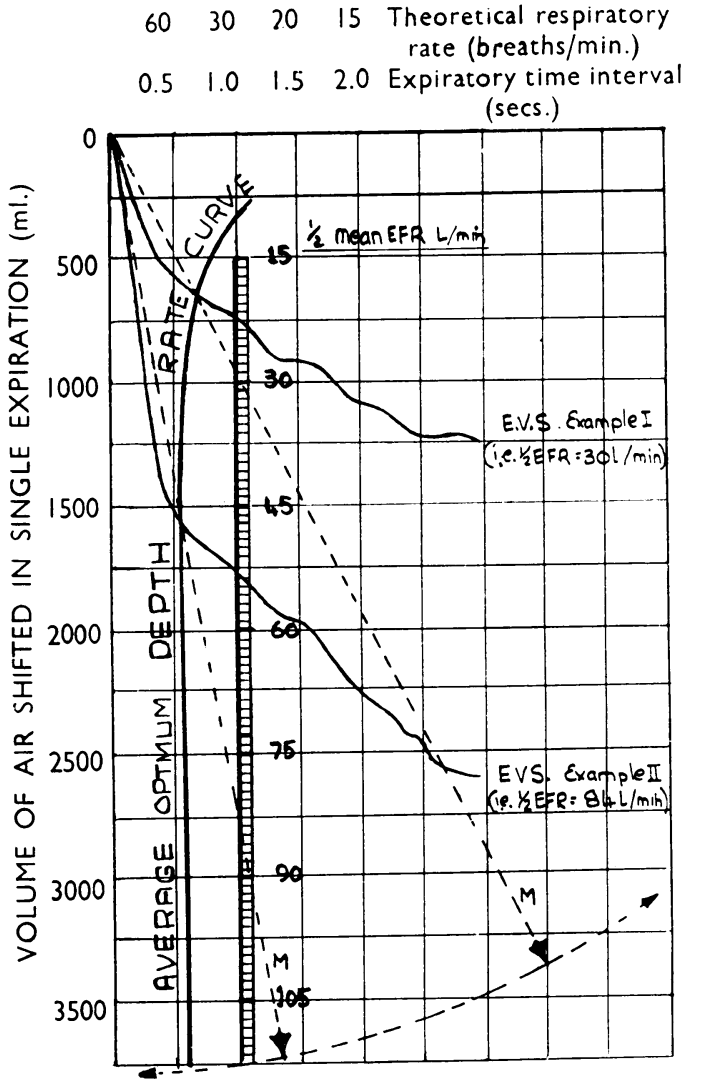

FIG. 9.-The transparent nomogram has a movable hair line $(m)$ with its centre of axis at zero. The nomogram is placed on the E.V.S. curve to be measured, the zero point being placed immediately over the point of origin of the curve, and the whole transparent nomogram aligned to the true vertical of the E.V.S. tracing. The point at which the E.V.S. cuts the average optimum depth rate curve can then be seen and the movable hair line is then 3 moved to cover the point of intersection. The point where the moved to cover the point of intersection. The point where the E.V.S. curve being measured. (Protractor A K 3.8/R.D.O.C.)

$60 \mathrm{l} / \mathrm{min}$. or less. It is the E.V.S. of these subjects that cuts the upper curved portion of the $\frac{D}{0}$ optimum depth rate line, and who have a higher M.V.V. than their expiratory flow rate. Conse- N quently if the optimum depth rate line be replaced $N$ by a fixed intercept line, which is equivalent to a breathing rate of 40 breaths per minute, then auto- $\omega$ matically the prolonged expiratory time interval of these subjects would be partially compensated. For the above reasons the mean expiratory flow rate was measured over the first $0.75 \mathrm{sec}$. period of the E.V.S. This measure has been abbreviated to E.F.R. ${ }^{40}$, since it is equivalent to a breathing $\underset{\mathbb{D}}{\overparen{D}}$ rate of $40 / \mathrm{min}$.

In practice six consecutive E.V.S. tracings are $\varrho$ recorded, and using a transparent perspex protractor (Fig. 12) the E.F.R. ${ }^{40}$ for each tracing is 


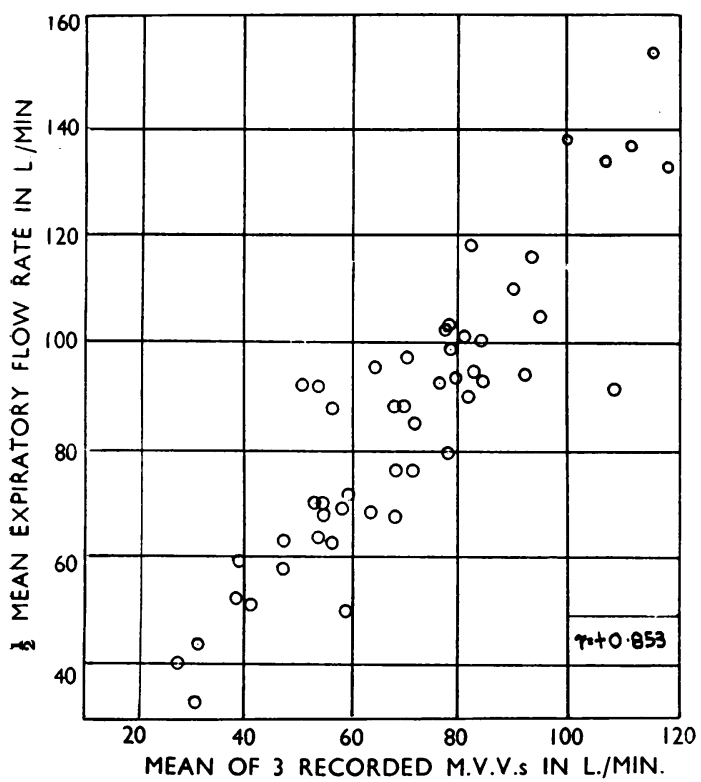

FIG. 10.-Expiratory flow rate values (mean of six E.V.S.), using the average optimum depth rate curve nomogram plotted against M.V.V.(50 subjects). Protractor A $/ K$ 3.8/R.D.O./C. was used

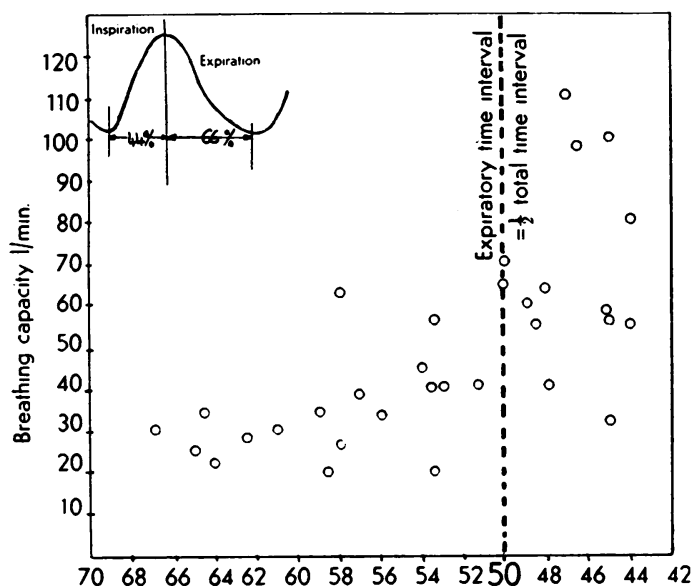

Fig. 11.-The expiratory : total respiratory time ratio of individual patients related to their M.V.V. The mean expiratory time interval of four M.V.V. tracings is expressed as a percentage of the total time interval.

measured. The mean of these six readings of the E.F.R. ${ }^{40}$, corrected to $37^{\circ}$ C. and saturated with water vapour, is taken as the E.F.R. ${ }^{40}$ of the subject.

In Fig. 13 the E.F.R. ${ }^{40}$ of 50 different subjects has been plotted against the recorded M.V.V. The correlation coefficient for these two different measures was +0.927 . In certain subjects the

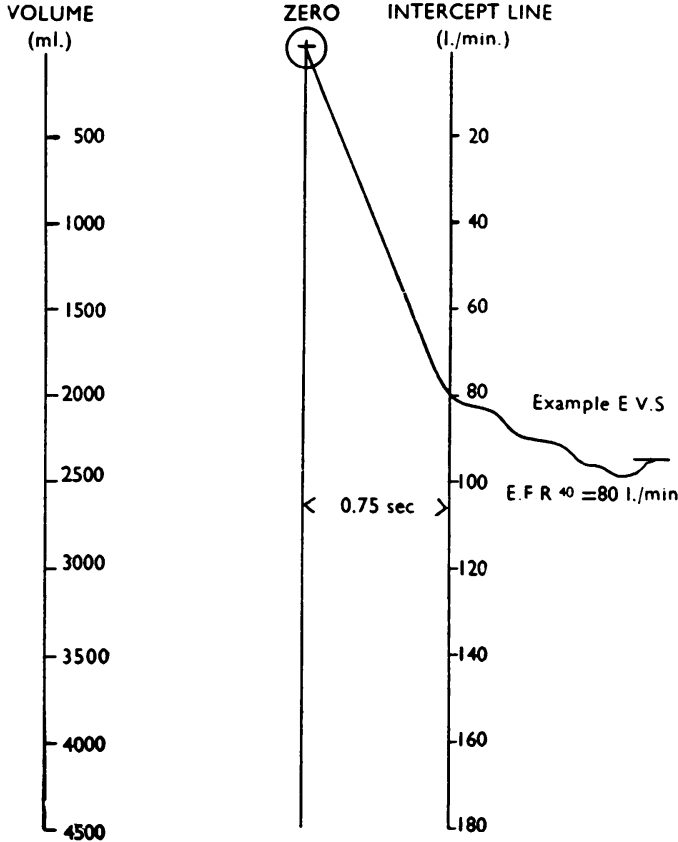

FIG. 12.-Diagram of the transparent protractor. Zero point in the centre of the circle is superimposed on the point of origin of the E.V.S. to be measured. The vertical lines on the protractor are next aligned with the true vertical lines on the tracing. The point aligned with the true vertical lines on the tracing. The point
where the E.V.S. cuts the intercept line gives the E.F.R.40 of where the E.V.S. cuts the intercept line gives the E.F.R.40 of
the E.V.S. (in the example above the E.F.R.40 is $801 . / \mathrm{min}$.) the E.V.S. (in the example above, the E.F.R.40 is $801 . / \mathrm{min}$.). uated according to the volume of the spirometer and the speed of the drum periphery of the kymograph respectively.

E.F.R. is considerably higher than the M.V.V., and it is suggested that most of these subjects did not give of their best when performing the M.V.V. test or that the M.V.V. test causes rapid fatigue so that a low value is obtained.

From the analysis of the tracings of 27 men it was found that the standard error of each individual tracing averaged $5.951 . / \mathrm{min}$. and the mean of six tracings of an individual, each measured once, had a standard error of $2.431 . / \mathrm{min}$. However, the variation from tracing to tracing was not constant but increased with the size of the reading. Ignoring the small error associated with the actual measurement with the protractor, the variation from tracing to tracing can be best expressed as a proportional standard error of 1.087 , so that $95 \%$ confidence limits for a single reading are then given by dividing and multiplying the reading by 1.182. Thus a reading of $66 \mathrm{l} / \mathrm{min}$. would have $95 \%$ confidence limits of $\frac{66}{1.182}=55.8$, and $66 \times$ $1.182=78 \mathrm{l} / \mathrm{min}$. If the mean of six tracings were 66 , the confidence limits for this mean would 


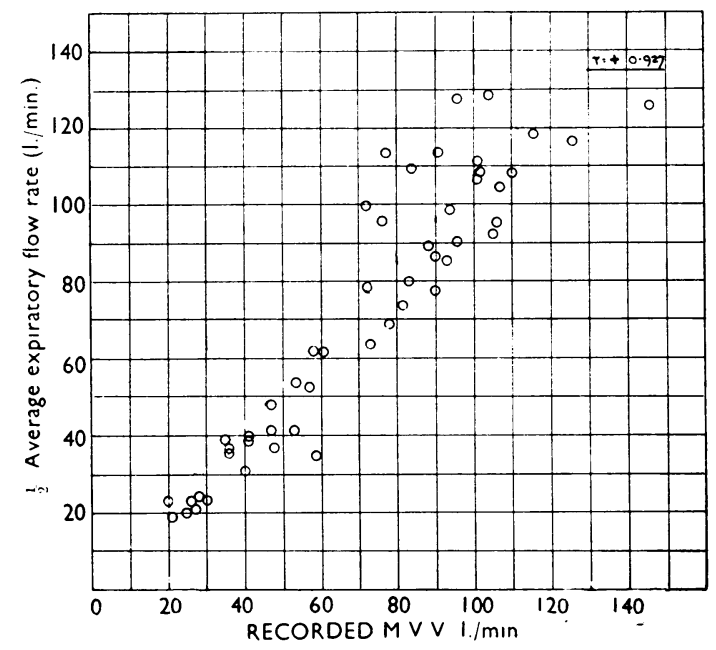

FIG. 13.-Half the average E.F.R. over the first $\frac{3}{4} \mathrm{sec}$. period of six consecutive E.V.S. tracings plotted against the mean of three assessments of the M.V.V. ( 50 subjects).

then be 62.9 and 69.3. The standard error of the mean of six readings is, therefore, roughly of the order of $5 \%$.

The E.F.R. over the first $1.5 \mathrm{sec}$. period (E.F.R. ${ }^{20}$ ) of the E.V.S. has also been compared with the M.V.V. in the same 50 subjects. In six of these subjects it was impossible to obtain a reading of the E.F.R., since the E.V.S. did not cover the 1.5 sec. time interval. In the remaining 44 subjects the E.F.R. has been plotted against the M.V.V. figures (Fig. 14), and although the correlation is good $(r=+0.913)$ this measurement of the E.F.R. (over $1.5 \mathrm{sec}$. of the E.V.S.) does not

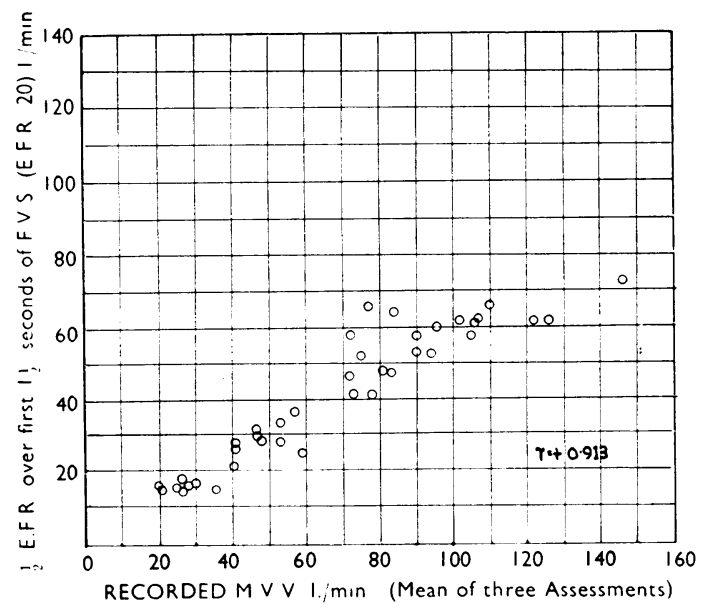

FIG. 14.-Expiratory air flow rates over the first $1 \frac{1}{2}$ sec. period of a forced expiration (theoretical respiratory rate $=20$ breaths $/ \mathrm{min}$.) plotted against recorded M.V.V.s. spread out the different subjects as well as the $0.75 \underset{\overrightarrow{\vec{m}}}{\overrightarrow{0}}$ sec. intercept line. Further, the E.F.R. measured over the first $0.75 \mathrm{sec}$. period of the E.V.S. (E.F.R. ${ }^{40}$ ) agrees closely with the M.V.V. values, and since the M.V.V. has been a standard measure $\vec{\nabla}$ for some time the E.F.R. ${ }^{40}$ intercept line has been $\stackrel{ }{0}$ adopted as the method of choice.

\section{Discussion}

By measuring half the mean expiratory flow ratect over the first $0.75 \mathrm{sec}$. period of six consecutive E.V.S. (E.F.R. $\left.{ }^{41}\right)$, a stable and repeatable measure ${ }^{x}$ of ventilatory function is obtained. The standard error of a single E.V.S. over a wide range of values measured in this way is $5.951 . / \mathrm{min}$., which compares favourably with that of a single assessment of the M.V.V., which has a standard error of 7.5 1./min. (Gilson and Hugh-Jones," 1949). By@ measuring the mean of six consecutive E.V.S.s the⿳⺈冂大 standard error is reduced to 2.431 . / min. Evidence $\vec{B}$ is given showing that the error varies with the E.F.R. values, and for practical purposes the standard error is taken as being $\pm 5 \%$. Thus the absolute standard error is less than $2.431 . / \mathrm{min}$. when the E.F.R. ${ }^{40}$ value is low.

The M.V.V. test is generally accepted as åू useful test of maximum ventilatory capacity, and $\stackrel{\mathbb{Q}}{\mathscr{Q}}$ from the results it can be seen that the E.F.R. ${ }^{41} \overrightarrow{\vec{B}}$ gives very similar values to the M.V.V. test. The 3 M.V.V. test has a number of practical limitations. In seriously ill patients it cannot be applied. A? high degree of co-operation from the subject is required. The test is fatiguing, time-consuming, and cannot readily be used for the hour-to-hour $\underset{x}{0}$ assessment of a subject during treatment. Theo E.F.R. ${ }^{40}$ test takes on an average two to four minutes to perform, depending on the incapacityô of the patient. This is less than half the time required for the M.V.V. test. Further, the sixo E.V.S. tracings, from which the E.F.R. ${ }^{4 i}$ is measured, provide a permanent record which cano be easily filed and compared quantitatively and qualitatively with later tracings.

In all these respects the E.F.R. ${ }^{40}$ test is prefer- 0 able to the M.V.V. test. It has been applied with success to many patients in status asthmaticus.o Satisfactory measures of the E.F.R. ${ }^{40}$ can beo obtained with very little co-operation from the sub-क ject, and, consequently, for the assessment for com? pensation of subjects with industrial lung lesionso the measurement of E.F.R. ${ }^{40}$ is preferable to the M.V.V. test. But if this test is to be used for the assessment of industrial lung lesions for com $\Phi$ pensation, it will be necessary to produce a table of E.F.R. values in normal subjects related to sex 
age, and anthropometric measurements. This has already been done with normal children, and the studies are being extended to normal adults.

In subjects whose expiratory:inspiratory time ratio is $1: 1$, the measurement of the expiratory flow rate over the first $0.75 \mathrm{sec}$. time period is equivalent to a theoretical breathing rate of 40 breaths per minute (E.F.R. ${ }^{40}$ ). This E.F.R. ${ }^{40}$ has been chosen as the measure of ventilatory capacity for the following reasons. First, if the E.F.R. be measured over shorter time intervals, the accuracy of the measure progressively diminishes. Second, it has been shown that the M.V.V. of a subject, as measured on the apparatus used, is influenced mainly by the tidal volume per breath. The optimum rate of breathing for most subjects performing the M.V.V. test is found to vary between 45 and 60 breaths per minute. This finding is substantiated from my observations of many hundreds of athletes and troops, exerting themselves to the limit of physical endurance, who do not, except very rarely, breathe faster than 40 breaths per minute ; in fact their respiratory rate under these conditions is usually between 35 and 40 breaths per minute. Robinson (1938) found that the respiratory rate of normal subjects during maximal work decreased with advancing age. Six adolescents with a mean age of 18 years had a mean respiratory rate of 46 breaths $/ \mathrm{min}$., while seven men whose mean age was 62.7 years had a respiratory rate of 35 breaths $/ \mathrm{min}$. Forty-seven subjects, aged 16 to 71 years with a mean age of 39 years, had a mean respiratory rate of 40.8 breaths $/ \mathrm{min}$. Third, there are good theoretical grounds for measuring the ventilatory capacity at a fixed respiratory rate. It is known that values for the anatomical dead-space air may vary in different diseases, and may increase in the same individual during hyperventilation (Rossier and Buhlmann, 1950). However, the proportion of a subject's actual ventilation which is ineffective on account of the dead-space air decreases as the tidal volume increases and as the respiratory rate decreases. This is theoretically shown in Fig. 15, where the anatomical dead-space air has been assumed to be constant at $150 \mathrm{ml}$. It can be seen that for any fixed respiratory rate the actual ventilation that is ineffective on account of the dead-space air remains constant whatever the tidal volume. For this reason the E.F.R. ${ }^{40}$ is preferable to the M.V.V. test, where the respiratory rate may vary considerably from patient to patient.

Much has been written concerning the relationship between the vital capacity and the maximum voluntary ventilation of a subject. Cournand and

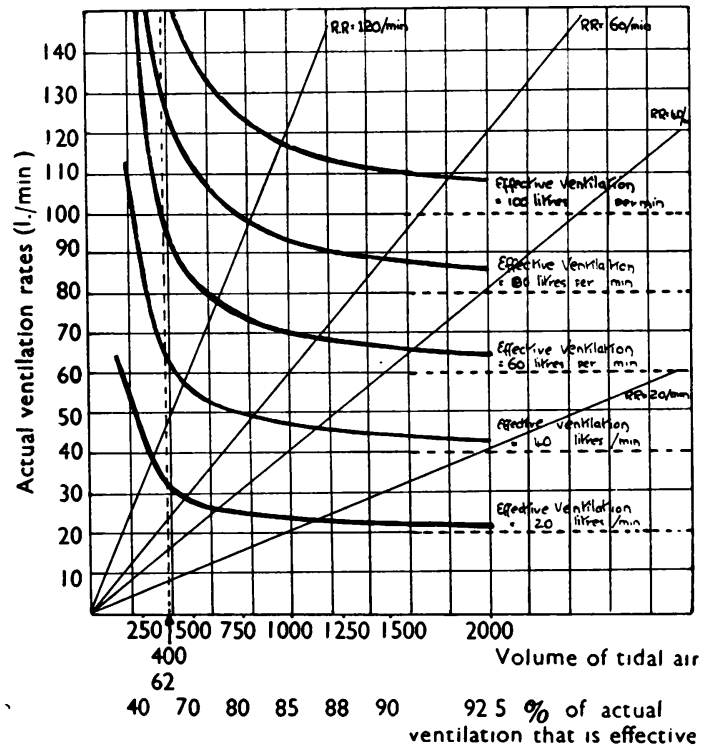

FIG. 15.-Theoretical graph based on the assumption that the deadspace air is constant at $150 \mathrm{ml}$. It shows the proportion of different ventilation rates that are ineffective on account of the dead-space air and how this proportion decreases as the tida volume increases and or the respiratory rate decreases. The five thick black lines indicate, from top to bottom, the actual five thick black lines indicate, from top to bottom, the actual $100,80,60,40$, and $201 . / \mathrm{min}$. respectively. R.R.= respiratory 100,
rate.

others (1939), studying 20 normal males and 20 normal females, found the correlation between vital capacity and M.V.V. to be +0.506 and +0.634 respectively. Gilson and Hugh-Jones (1949), studying a series of 17 normal and pneumoconiotic subjects, found a correlation coefficient of $r=+0.826$, and they concluded that this relationship was largely due to the high correlation between complementary air and M.V.V. $(r=+0.854)$. They also found that two men with the same vital capacity may have M.V.V.s varying by as much as $801 . / \mathrm{min}$. It has been shown in this paper that the E.F.R. ${ }^{40}$ of 50 subjects correlates closely with the observed M.V.V. ( $r=+0.927)$ and also that the volume expelled over the first $0.75 \mathrm{sec}$. period of a full expiration would show the same relationship. These findings show in general that no useful purpose is served by attempting to predict the M.V.V. from the whole of the vital capacity, since the vital capacity of an individual is only a true measure of ventilatory capacity when most of the vital capacity can be expelled at a uniform rate and when this rate is proportional to the vital capacity.

Other authors have also been using the first portion of single vital-capacity tracings for assess- 
ing maximum voluntary ventilation. In France, Roche and Thivollet (1949) and Tiffeneau, Bousser, and Drutel (1949) measured the volume of air that is expelled in the first second of a forced maximum expiration. Tiffeneau and others termed this measure "la capacité pulmonaire utilisable à l'effort" (C.P.U.E.). However, by using a onesecond time interval they are cutting the E.V.S. at a level considerably lower than the critical point (see Fig. 1). Guillet (1951) has measured the M.V.V. indirectly by converting the C.P.U.E. volume of Tiffeneau into units of flow by multiplying the C.P.U.E. by 30 . He found this indirect assessment of the M.V.V. and the direct assessment of the M.V.V. to be similar in 118 cases of silicosis. However, he concluded that the indirect assessment of the M.V.V. was preferable to the direct method, since it was simple and practical, and separated the radiological disease groups of silicosis better. Hanaut (1953) has recently published normal values of this indirect assessment of the M.V.V. related to age and anthropometric measurements. Gaensler (1951) measures the volume of air expelled in one second and terms it the "one-second capacity," which is recorded directly on a dial. Although this method may be slightly more convenient in practice, no tracings are obtained. The E.V.S. tracings described above are remarkably constant and almost specific for a given individual at any particular time, though they may vary from day to day if there is any change in the factors influencing air flow. Thus, it is usually easy to see from the tracings whether a patient is co-operating. For this reason there is an advantage in recording tracings rather than dial readings. In Czechoslovakia, Kadlec and Vyskocil (1950) have measured the $V$ and $Y$ components of the first fraction of forced vital-capacity tracings as defined in this communication, and have used an arbitrary index (I.V.C.) given by the formula I.V.C. $=2 \frac{\text { V.C. }}{\mathbf{P}}-\frac{\text { V.C. }}{\mathrm{V}}-\mathrm{Y}$, where V.C. and $\mathrm{V}$ are measured in litres, $Y$ in seconds, and $P$ is the body surface area. Further, they suggested that in the assessment of disability in cases of silicosis the I.V.C. index was preferable to the M.V.V., since it discriminated better between normal and abnormal ; it was simpler and quicker to carry out and was not so dependent on the co-operation of the patient.

The value of the E.F.R. ${ }^{40}$ measure as an index for assessing bronchodilator drugs has already been discussed (Kennedy and Stock, 1952). Normal values of the E.F.R. ${ }^{40}$ related to age, sex, and anthropometric measurements, and the diagnostic value of the E.V.S. in different diseases, will be presented in a future paper.

\section{Conclusions and Summary}

The vital capacity, recorded on a fast-revolving $\stackrel{\frac{6}{\overparen{\Phi}}}{\overparen{D}}$ drum during expiration and inspiration, has been $\mathcal{O}$ termed the vital spirogram. It is shown that the $e^{\infty}$ expiratory phase of this vital spirogram (E.V.S.) $\overrightarrow{0}$ has an initial linear fraction, the volume and rate of air flow of which is clearly related in shape to $\vec{\omega}$ that seen during the expiratory phase of maximum voluntary ventilation (M.V.V.). It is shown that the slope of the first steep fraction of the E.V.S. correlates well with the expiratory phase of the M.V.V. tracing in 27 patients $(r=+0.975)$. Con- 0 secutive E.V.S. tracings are remarkably constant ${ }^{\supset}$ for a given individual for an occasion, though they $\vec{z}$ may vary from day to day if there is a change in the factors influencing air flow. The tidal volume $\widehat{\supset}$ and rate of breathing during voluntary hyperventilation have been studied in 332 potteryo workers and Cornish tin-miners. It was found that these subjects breathed the greatest volume of airo in unit time at a respiratory rate of between 45 and 55 breaths per minute. Their maximum ventil-o atory capacity, therefore, depended on their tidalo volume when breathing between these rates.

Half the mean expiratory flow rate over the firsto $0.75 \mathrm{sec}$. period of six consecutive E.V.S. tracings 3 has been termed the E.F.R. ${ }^{40}$, since the $0.75 \mathrm{sec}$. period is equivalent to a theoretical breathing rate of 40 breaths per minute. The E.F.R..$^{40}$ is readily obtained from the E.V.S. tracings by the use of a을 transparent protractor.

In 50 patients with chronic lung diseases the correlation coefficient of the E.F.R..$^{40}$ test and the M.V.V. test was +0.93. The E.F.R. ${ }^{40}$ test is aO better measure of the maximum ventilatory capacity than the M.V.V. test, because it is easier and quicker to do and has a small standard error (S.E. $= \pm 5 \%$ ).

It is suggested that the measurement of ventil $N$ atory rates, using the E.F.R. ${ }^{40}$ test, should provo a useful method of estimating the maximum ventil 군 atory capacity of individuals in hospital and field work, and especially for the assessment for com pensation of patients with pneumoconiosis.

The vital capacity is only a valid measure of the maximum ventilatory capacity when most of the vital capacity is expelled at a uniform rate ano when this rate is proportional to the vital capacity The E.F.R. ${ }^{40}$ is a measure of that part of the vita? capacity which is of use to the subject for hyper ventilation. 
I am indebted to Dr. J. C. Gilson, Mr. P. D. Oldham, and Dr. J. P. P. Stock for advice and criticism; to Mr. T. E. Bromley, S.R.N., for carrying out most of the spirometry ; to Mrs. E. B. I. Williams for measuring most of the tracings.

\section{REFERENCES}

Baldwin, E. de F., Cournand, A., and Richards, D. W. (1949). Medicine, 28, 1 .

Cournand, A., Richards, D. W., and Darling, R. C. (1939). Amer. Rev. Tuberc., 40, 487

Ferris, B. G., Affeldt, J. E., Kriete, H. A., and Whittenberger, J. L. (1951). Arch. industr. Hyg., 3, 603.

Gaensler, E. A. (1951). Amer. Rev. Tuberc., 64, 256.

Gilson, J. C., and Hugh-Jones, P. (1949). Clin. Sci., 7, 185.

(1953). To be published.
Gray, J. S., Barnum, D. R., Matheson, H. W., and Spies, S. N. (1950). J. clin. Invest., $29,677$.

Guillet, M. (1951). La Place de la Spirographie dans l'Expertise en Matiere de Silicose. Imprimerie Grandville, Nancy.

Hanaut, A. (1953). Proc. 10th Int. Congr. on Industr. Med., 1951. Lisbon. To be published.

Hermannsen, J. (1933). Z. ges. exp. Med., 90, 130.

Kadlec, K., and Vyskocil, J. (1950). Pracovni Lekarstvi, 6. 348.

Kennedy, M. C. S. (1950). Beitr. Silikose-Forsch., Heft 1C, p. 19.

and Stock, J. P. P. (1952). Thorax, 7, 43.

Robinson, S. (1938). Arbeitsphysiologie, 10, 251.

Roche, L., and Thivollet, J. (1949). Arch. Mal. prof., 10, 448.

Rossier, P. H., Bucher, H., and Wiesinger, K. (1947). " Uber die Silikose," p. 83. Vjschr. Naturf. Ges. Zurich, 92, Beihefte 3-4. _ and Buhlmann, A. (1950). "Uber die Silikose," pt. 2, p. 51. Ibid., 95, Beihefte 2-3.

Tiffeneau, R., Bousser, J., and Drutel, P. (1949). Paris med. (partie méd.), 39, 543. 